\title{
Difference in taste identification among obese and non obese adult men
}

\begin{abstract}
Background: It has already been studied in past in different countries excluding Pakistan, that Obese and non-obese children and adolescents differ in their taste perception. However, this has predominantly been investigated for children and adolescents and data are partly in consistent and insufficient when it comes to adults. Therefore, this study has been taken.
\end{abstract}

Objectives: The aim of the study was to determine differences in taste identification between obese and non-obese adult males.

Methodology: In an experimental study of $\mathrm{n}=25$ obese males and non-obese of ages $>30$ years, taste identification of four taste qualities (sweet, sour, bitter and salty) was compared. Taste strips impregnated in taste solutions were used for gustatory testing. Subjects were asked to rank the intensity of the taste quality, on a 5 point rating scale. Chi square was used to analyze the data.

Result: There was a significant difference in sweet taste quality ( $p$ value 0.009 ).Obese subjects $(60 \%)$ compared to the non-obese $(92 \%)$ showed lower ability to identify the sweet taste quality. However, marked difference was noticed in other three qualities i.e. bitter $($ obese $=32 \%$, non-obese $=40 \%)$, sour $($ obese $=44 \%$, non-obese $=56 \%)$ and salty (obese $=68 \%$, non-obese $=76 \%$ ) but it was not statically proven $(\mathrm{p}=>0.05)$

Conclusion: Obese and non-obese males differ in their taste identification. Obese males could identify less precisely than non-obese participants.
Volume 6 Issue 3 - 2017

\author{
Mahira Khan,' Hina Qazi \\ 'Darul sehat hospital, Pakistan \\ ${ }^{2}$ Nutrition and Dietetics Department, Pakistan
}

Correspondence: Mahira khan, Bachelors in Nutrition, Ward dietitian at Darul sehat hospital, Flat G-214, Gulshan-e-shamim, Karachi, Pakistan, Email mahirakhan593@gmail.com

Received: January 28, 2017 | Published: March 16, 2017

\section{Introduction}

The prevalence of obesity has grown in parallel with the worldwide becoming a global public health problem that threatens the economies of all nations. Obesity is considered as a disease caused by a multi factorial etiology that has a genetic basis, but requires lifestyle influences to manifest. ${ }^{1}$ Obesity is fuelled by individual factors, nutrition transition and increasingly sedentary lifestyles that lead to excess caloric intake. ${ }^{2}$ Taste is a primary aspect by which children determine food acceptance. It plays an essential role in eating behavior. ${ }^{3}$ In an evolutionary context taste has an important function in the identification of valuable nutrition: sweet tastes promise readily available calories whereas bitter often indicates toxic substances.

Taste sensitivity varies between individuals. Several studies describe differences between obese and non-obese subjects concerning their taste perception. It has been suggested that people with high BMI may have a higher motivation to eat and less motivation to cease eating when they are paying attention to taste. ${ }^{4}$ This implies that a change in taste perception, which may alter attention to a particular taste, may affect eating behavior in groups with different BMIs. Gender differences have been reported by several authors. Cohen \& Gitman $^{5}$ found that men had a higher incidence of taste errors than women when they had to recognize the basic tastes of sour, sweet, salty and bitter.

It has already been studied in past in different countries excluding Pakistan, that Obese and non-obese children and adolescents differ in their taste perception. However, this has predominantly been investigated for children and adolescents and data are partly in consistent and insufficient when it comes to adults. Therefore, this study has been taken in order to find out the differences in taste identification between obese and non-obese adult males.

\section{Methodology}

This type of research is experimental and qualitative in nature. The population consisted of adult men of age $>30$ years living in Karachi. The convenient sample was collected and its total size was 50 adult men i.e. 25 obese and 25 non-obese. Taste sensitivity was the dependent variable whereas, BMI was independent variable.

\section{Tools for data collection}

Observation sheet and taste strips were used as a tool for data collection. Observation sheet included anthropometric measurements (height \& weight) and a 5-point scoring scale was developed for comparing the intensities of all four tastes with 1 , representing No Taste, 2 representing slight, 3 representing moderate, 4 representing Strong and 5 representing very strong taste. Basic idea about this rating scale was developed from a previous research. ${ }^{6}$ Taste strips were made of filter paper soaked in taste solutions for $1 \mathrm{~min}$ and was dried.

a. Sweet: table sugar: 1 teaspoon dissolved in 2 tablespoons water

b. Salt: table salt: 1 teaspoon dissolved in 2 tablespoons water

c. Sour: lemon: 1 teaspoon dissolved in 2 tablespoons water

d. Bitter: PROP (propylthiouracil): $1 / 2$ tablet dissolved in 2 tablespoons water

The basic idea of the taste strips was developed from a previous class experiment. ${ }^{7}$ 


\section{Methods of data collection}

Body weight and Height was measured and BMI was calculated. Asian cut-offs were used for identifying obese (BMI $>25)$ and nonobese $(\mathrm{BMI}<24.9)$ men. Taste strips were placed on the tongue and subjects were asked to identify the taste quality by choosing one of four possible answers (sweet, sour, salty, bitter). Before assessment of each taste strip the mouth was rinsed with water. Subjects were asked to rank the intensity of the taste quality; ranked on a 5-point rating scale

\section{Statistical methods}

The level $\mathrm{P} \leq 0.05$ was considered as the cut-off value for significance. Data were presented using descriptive statistics and Chi square test was used to analyze the association of taste identification with BMI. The same test was used for the comparison of intensities of all four tastes (sweet, bitter, sour and salty).

\section{Results of the study}

\section{Characteristics of the subject}

The population consisted of adult men of age $>30$ years. The convenient sample was collected from people living in Karachi. The total sample size was 50 adult men i.e. 25 obese and 25 non-obese. Table 1 indicates the average age, height, weight and BMI of the subjects. The findings of the Table 1 show that most of the participants were in their 40's.

Table I Clinical characteristic of the Participants

\begin{tabular}{lll}
\hline \multirow{2}{*}{ Average } & \multicolumn{2}{l}{ BMI ranges } \\
\cline { 2 - 3 } & Obese & Non-obese \\
\hline Age (years) & 44.9 & 44.4 \\
Weight $(\mathrm{kg})$ & 82.98 & 65.8 \\
Height (meters) & 1.67 & 1.7 \\
BMI $\left(\mathrm{kg} / \mathrm{m}^{2}\right)$ & 29.8 & 22.3
\end{tabular}

\section{Differences in the identification of all taste qualities}

The data was collected through observation sheet to evaluate the differences in taste identification among obese and non-obese adult men. Chi square was use to analyze the association of taste identification with BMI. To illustrate the differences in identification of taste, data was presented graphically to show the percentage of all taste qualities identified by obese and non-obese males.

Sweet taste: Obese subjects had difficulty in identifying the correct taste quality as compared to the non obese subjects' shows Figure 1. There was a significant difference in sweet taste identification between obese and non obese adult males ( $p$ value 0.009 ).

Bitter taste: Marked difference had been shown in identifying the correct taste quality between obese and non obese males shows Figure 2 but, it was not statistically proven (pvalue 0.384 ).

Sour taste: Most of the non obese males were able to detect the correct taste quality shows Figure 3, however No statistically proven difference had been shown in identifying the sour taste quality ( $p$ value 0.286 ).

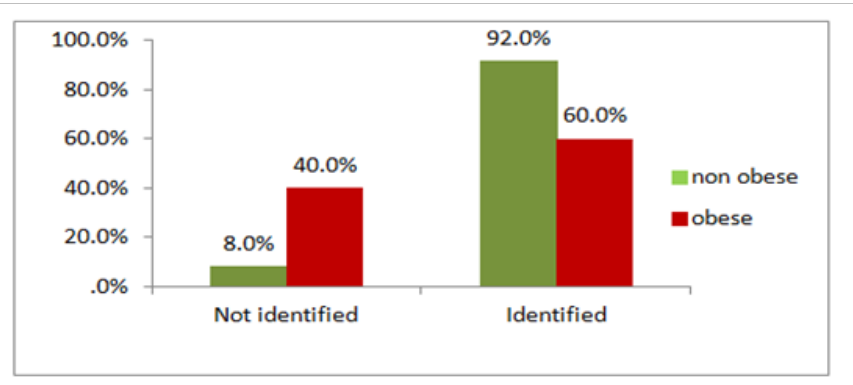

Figure I Differences in sweet taste identification.

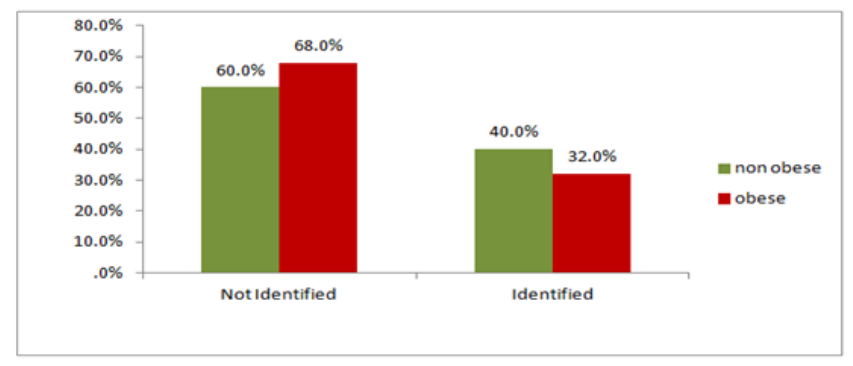

Figure 2 Differences in bitter taste identification.

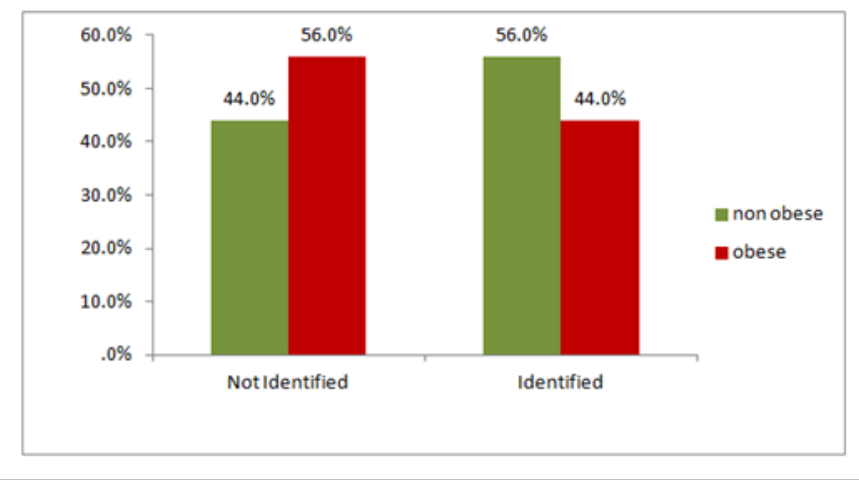

Figure 3 Differences in sour taste identification.

Salty taste: When scrutinizing, the result shows no statistically proven difference in identification of salty taste ( $\mathrm{P}$ value 0.377 ) however noticeable difference had been displayed in Figure 4.

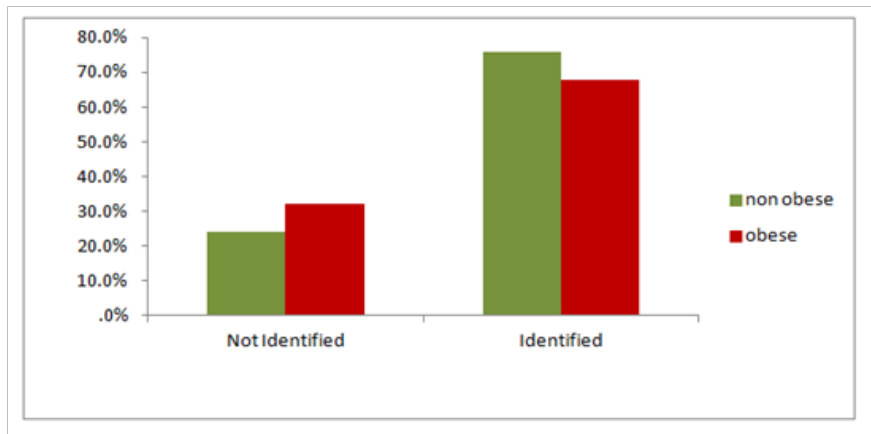

Figure 4 Differences in salt taste identification.

\section{Relationship of intensity rating with weight}

Chi square was use to look over the interrelation of intensity ratings with BMI. To interpret the differences in intensity of taste, data was presented graphically to show the percentage of all taste qualities. 
Intensity rating for the sweet taste quality: In the sweetness intensity rating, obese $(n=25)$ and non-obese $(n=25)$ adult males rated sweet strips according to their intensity. Most of the obese group rated lower on the intensity scale when compared to the non-obese participants' shows Figure 5. However, it was not statistically proven $(p=0.943)$

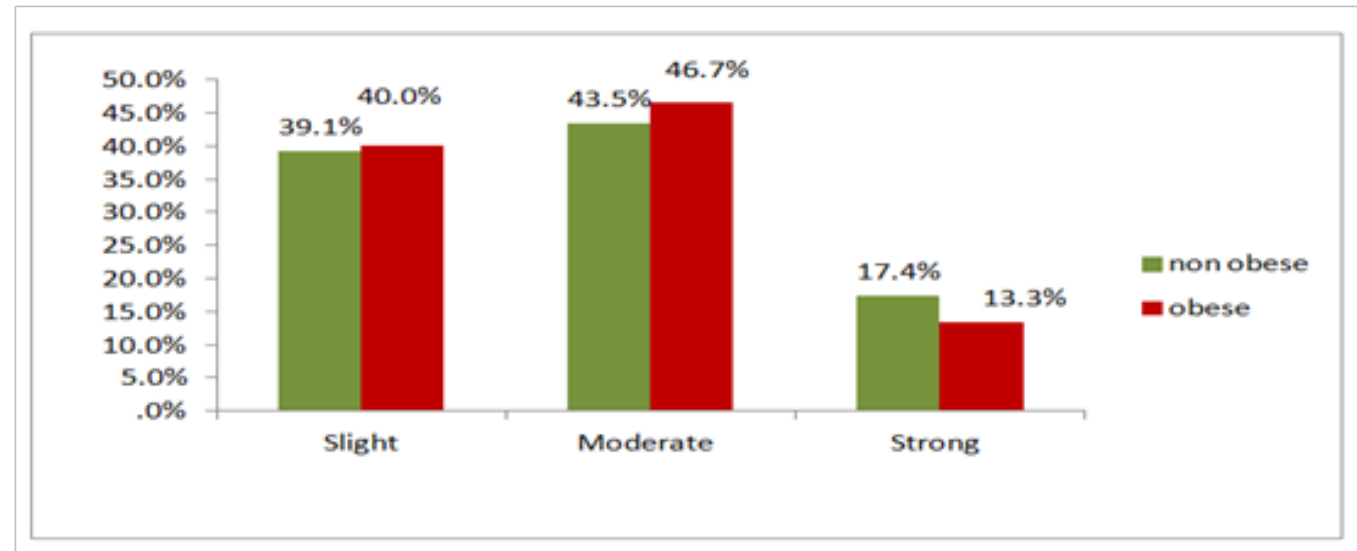

Figure 5 Differences in intensity of sweet taste.

Intensity rating for the bitter taste quality: When examining the bitter intensity rating, it was found out that most of the non-obese group rated higher intensities when compared to the obese groups show Figure 6 , but it was not statistically proven $(\mathrm{p}=0.771)$.

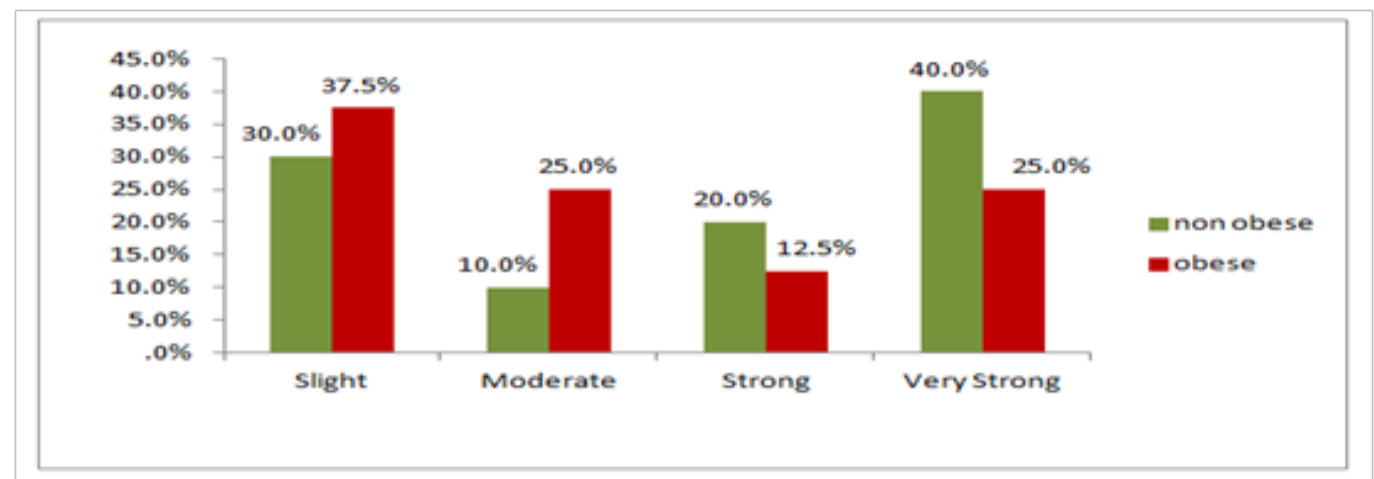

Figure 6 Differences in intensity of bitter taste.

Intensity rating for the sour taste quality: There was a modest difference in the intensity of sour taste quality when compared. Most of the obese and non-obese participants rated slight intensity more often shows Figure 7, although the results are not statistically proven $(\mathrm{p}=0.955)$.

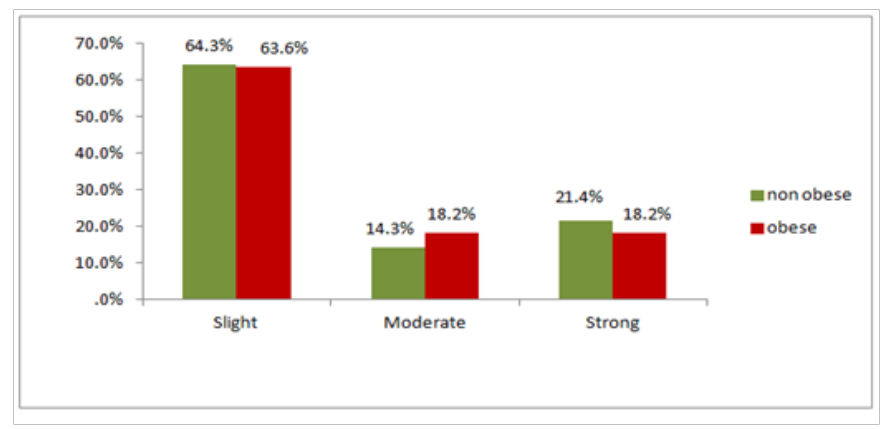

Figure 7 Differences in intensity of sour taste.

Intensity rating for the salt taste quality: No statistically proven difference had been shown in the intensity of salt taste quality $(\mathrm{p}=0.110)$, nonetheless majority of the non-obese rated higher intensities of salty taste when compared to the other group illustrates Figure 8.

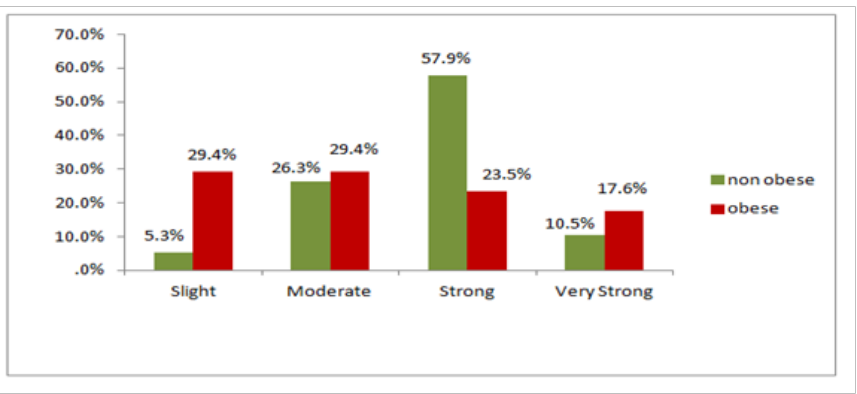

Figure 8 Differences in intensity of salt taste.

\section{Classification of respondents on the basis of total score}

The sum of all four possible taste qualities (sweet, bitter, sour and salt) resulted in a maximum total score of 4 . On the basis of the total score, respondents are designated into poor, average and super tasters. The outcome reveals that most of the non-obese participants fall in the average tasters on the other hand, most of the obese group falls in poor 
tasters when compared to the non-obese groups displayed in Figure 9, But the results are not statistically supported $(\mathrm{p}=0.094)$.

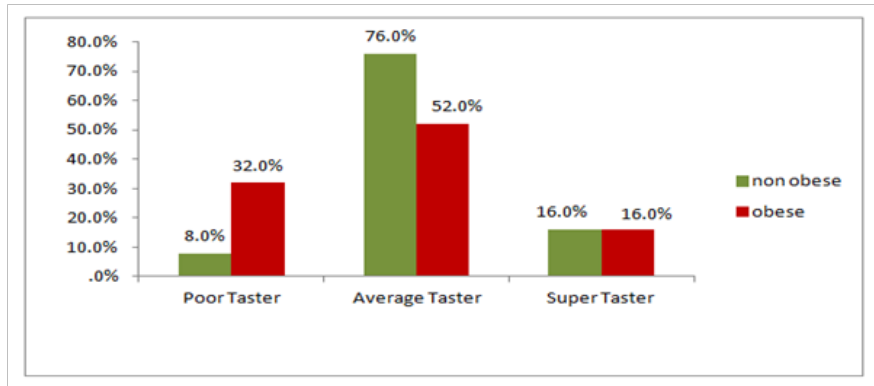

Figure 9 Classification of respondents on the basis of taste identification.

\section{Discussion}

The purpose of the study was to analyze difference in taste identification among obese and non-obese adult men (aged $>30$ years). Prevalence of obesity is increasing worldwide. It is considered a disease caused by a multifactorial etiology that has a genetic basis, but requires lifestyle influences to manifest. Worldwide, at least 2.8 million people die each year as a result of being overweight or obese. ${ }^{8}$ In contrast to the majority of studies concerning weight and status which had been taken place particularly on children and adolescence, data is insufficient when it comes to adults. Taste sensitivity varies between individuals. Several studies describe differences between obese and non-obese subjects concerning their taste perception.

The hypothesis that obese and non-obese adult males differ in their taste identification was confirmed in the present investigation. The findings of this research are somewhat similar to the previous researches done by Overberg J et al. ${ }^{9}$ They found out that Young obese subjects were found to have a lower ability to identify the correct taste qualities and rated sweetness lower in intensity compared to their normal-weight peers. ${ }^{9}$ In our study, the outcome reveals that obese subjects find it difficult to identify the sweet taste quality as compared to the non-obese peers which was also statistically supported. The authors explain that the reasons for these differences in taste sensitivity are poorly understood and most likely multi factorial, including genetic, hormonal and learning factors. ${ }^{10}$

Regarding the outcomes of total scores in the present research, obese subjects had a significantly lower total score in identifying the correct taste quality compared to their non-obese peers. Also, most of the obese subjects are poor tasters showed our investigation. Putting the results of previous research into context it reveals that, Obese subjects had a significantly lower total score in identifying the correct taste quality compared to their normal weight peers with respective scores of $12.6 \pm 3.0$ versus $14.1 \pm 3.0$ (out of a maximum of 20 points). Psychologist Linda Bartoshuk and her colleagues ${ }^{6}$ first coined the term "supertaster" in the 1990s. In their research, they noticed that some people seemed to have a much higher taste response than others. Women are much more likely to be supertasters than men ( 35 per cent of women vs 15 per cent of men) and more Asians are supertasters than the rest of the world. The outcomes of intensity rating in the present research showed that most of the obese groups rated lower on their intensity scale when compared to the non-obese individual. However, the data was not statistically proven but it showed a marked difference between the two groups. Unlike previous research which displayed that concerning the sweet intensity rating obese children gave significantly lower intensity ratings.

The differences in the present (adult men $>30$ years) and the previous research (children and adolescence) was because the subjects of the study were different. However, more researches are required to confirm these studies. Eventually, this could help develop further strategies of obesity prevention and therapy in adults.

\section{Conclusion}

To conclude, the result of the study supports the hypothesis that there is a difference in taste perception with increasing BMIs. As the current study is on a small scale with limited time, also, the prevalence of obesity is rapidly increasing; there is a need to conduct more relevant researches to find out the etiology of this multifactorial disease.

\section{Acknowledgements}

None.

\section{Conflict of interest}

The author declares no conflict of interest.

\section{References}

1. Qi L, Cho YA. Gene-environment interaction and obesity. Nutr Rev. 2008;66(12):684-694.

2. French SA, Jeffery, Story M, et al. Environmental influences on eating and physical activity. Rev Public Health. 2001;22:309-335.

3. Drewnowski A. Taste preferences and food intake. Аnпи Rev Nutr. 1997; 17:237-253.

4. Fernstrom JD. Introduction to the symposium. The roles of glutamate in taste, gastrointestinal function, metabolism, and physiology. Am J Clin Nutr. 2009;90(3):705S-706S.

5. Cohen T, Gitman L. Oral complaints and taste perception in the aged. $J$ Gerontol. 1959;14:294-298.

6. Anliker JA, Bartoshuk L, Ferris AM, et al. Children's food preferences and genetic sensitivity to the bitter taste of 6-n-propylthiouracil (PROP) Am J Clin Nutr. 1991;54(2):316-320.

7. Institute of Food Research. Taste and Flavour. Our Chemical Senses: Taste Test Your Taste. 2015. p. 1-12.

8. WHO. Global Health Observatory (GHO) data. 2015.

9. Overberg J, Hummel T, Krude H, et al. Differences in taste sensitivity between obese and non-obese children and adolescents. Arch Dis Child. 2012;97(12):1048-1052.

10. Kate Hilpern. Taste the difference: How our genes, gender and even hormones affect the way we eat. 2010. 